\title{
A numerical procedure to design internal cooling of gas turbine stator blades
}

\author{
Carlo Carcasci, Bruno Facchini
}

DEF, S Stecco Department of Energy Engineering, University of Florence, via di Santa Marta, 3-50139, Florence, Italy

(Received 7 November 1995; accepted 8 April 1996)

\begin{abstract}
Summary - The continuing need to improve both the efficiency and the specific power of gas turbines requires to progressively increase the temperatures of the turbine inlet.

Because the first stator blades are heavily thermally loaded, efficient blade cooling is necessary.

The cooling system is particularly delicate and its design must follow these guidelines:

- minimum thermodynamic and fluid dynamic losses;

- limited blade temperature even for reduced cooling mass flow.

Although the problem is important, analyses of possible designs are not common in literature and many constructors refer to practical experience and to various experimental results.

This paper presents a comparative investigation to determine the effects of internal and external cooling in the same blade, on the basis of different combined solutions as it often happens. The cooling model will be considered one-dimensional: the limitation in the accuracy of the results is by far overcome by the simplicity and versatility of the approach. Finally, practical hints for designing an effective cooling system are derived, with particular attention to impingement. Then, global cooling parameters and medium blade will be determined in off-design condition.
\end{abstract}

Résumé - Modélisation d'un système de refroidissement d'aubage de turbine à gaz. La nécessité d'accroître la puissance et le rendement des turbines à gaz requiert une élévation régulière de la température maximale du cycle.

Les sollicitations thermiques étant très fortes, le refroidissement de l'aubage est indispensable.

Le système de refroidissement à l'étude est très délicat et doit parvenir à ces résultats :

- contenir les pertes thermo-fluidodynamiques,

- garantir un bon fonctionnement même avec un débit réduit du fluide de refroidissement.

Dans cet article, nous présentons une étude comparative des différentes solutions possibles pour le refroidissement d'un aubage de turbine à gaz pour application industrielle. Les résultats montrent la possibilité de calculer la distribution de la température sur l'aubage afin d'optimiser sa répartition ainsi que son comportement en condition de charge partielle.

\section{Nomenclature}

\section{A section}

$A_{0}^{*} \quad$ equivalent hole area $\left[A_{0}^{*}=\left(\pi \cdot d^{2}\right) /\left(4 \cdot x_{n} y_{n}\right)\right]$

$b$ length

$B$ integration constant

$C_{D}$ flow coefficient

c pressure loss coefficient

$c_{p} \quad$ specific heat at constant pressure

$d$ diameter

$E \quad$ geometric parameter of impingement

$f$ friction coefficient

$G_{c}$ cross flow mass velocity $\left(G_{c}=m_{c} / A\right)$ (specific mass flow)

$G_{J}$ jet mass velocity $\left(G_{J}=m_{J} / A\right)$ (specific mass flow)

$h \quad$ specific enthalpy

$H$ heat exchange coefficient

$k$ number of control volumes

$K$ matrix element mass flow rate geometric constant cooling mass flow rate $\left(M_{f}=\rho_{c} u_{c} / \rho_{g} u_{g}\right)$

Mach number number of constant geometric zones pressure pressure upstream of impingement jet exchanged heat gas constant s-coordinate equivalent slot Stanton number temperature velocity direction hole pitch in cross flow direction pin fin pitch in coolant flow direction hole pitch in main flow direction channel height 


\section{Greek symbols}

$\beta_{p} \quad$ characteristic film cooling parameter $\left(x / S M_{f}\right)_{p}$

$\varepsilon_{h} \quad$ internal cooling efficiency

$\phi \quad$ dimensionless temperature gradient

$\rho \quad$ fluid density

$\eta \quad$ effectiveness

$\eta_{p} \quad$ maximum effectiveness value

$\eta_{f}^{*} \quad$ global film cooling effectiveness

$\mu$ dynamic viscosity

$\sigma \quad$ mean square root of temperature distribution

$\Omega_{c} \quad$ coolant surface

$\Omega_{g} \quad$ hot gases surface

Subscripts and superseripts

a adiabatic

c cooling / cross flow

ex exit

$g$ hot gases

$i$ index

in entrance

$j$ index

$J \quad$ jet

$\max$ maximum

min minimum

$p \quad$ peak

tot total

$w \quad$ wall

0 total quantity

* based plate or equivalent opposing heat transfer surface area

\section{INTRODUCTION}

Engine performance is determined by its efficiency and specific power. Both are closely related and, except in a few cases, they are likely to keep on improving as time goes on.

In a real gas turbine cycle, one could demonstrate that the efficiency and specific power are positively influenced by the increase in the ratio of the maximum to the minimum temperature of the cycle (Stecco and Facchini [16]; Carcasci [6]). The increase in the maximum temperature must correspond to an increased resistance to high temperatures of the materials used on the parts most exposed to the hot gases, especially the nozzles and the rotor blades in the first turbine stages. But this increased resistance being generally unsatisfactory, it is also necessary to cool adequately the most exposed parts (Stecco and Facchini [16 to 19]; Carcasci [6]).

Blade cooling reduces the advantages of the increased maximum temperature and, because the coolant is the air from the compressor, the efficiency begins to decrease above a certain temperature for a pre-established cooling system [16 to 19]. By improving the designs of the cooling systems, together with the heat resistance of the material, it is possible to limit these losses and thus to raise the maximum temperature levels and gain real increases in the efficiency and specific power of gas turbine plants.

In order to design cooling systems correctly, it is important to determine the surface blade temperature distribution. Therefore, it would be best to improve the efficiency of both the internal and external cooling and to try to obtain a uniform temperature distribution and a good internal and external cooling efficiency.

A typical cooling system combines different solutions both inside and outside the blade. Cooling systems are usually studied using an experimental approach which allows for the formulation of some correlations. In this field, various papers analyzing single cooling systems are available. This study will present a method for applying correlations and experimental results to a stator blade with combined cooling systems. The code developed is called COOL. In this work, the internal cooling systems described are:

- impingement, which consists of a series of jets of coolant against the inner surface of the blade; the jets are produced through a perforated layer within the blade itself (fig 1);

- pin fins, consisting of a series of small cylinders with their axes orthogonal to the coolant flow;

- tubes, a simplified cooling system at the trailing edge;

- as an external cooling system, only film cooling is examined; it consists in creating a film coolant surrounding either partially or completely the external surface of the blade, thereby protecting it from hot gases (fig 2).

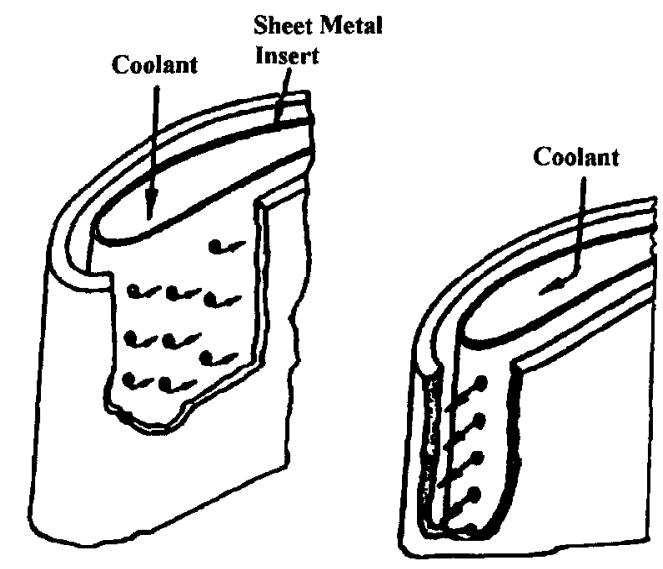

Fig 1. Impingement cooling system

The approach used for external blade temperature calculation comes from Arnone, Benvenuti et al [3] who proposed a numerical procedure for the evaluation of the heat coefficient in two-dimensional Navier-Stokes calculation of hot gas flow expansion.

The aim of this work is therefore the evaluation and completion of internal and external heat 


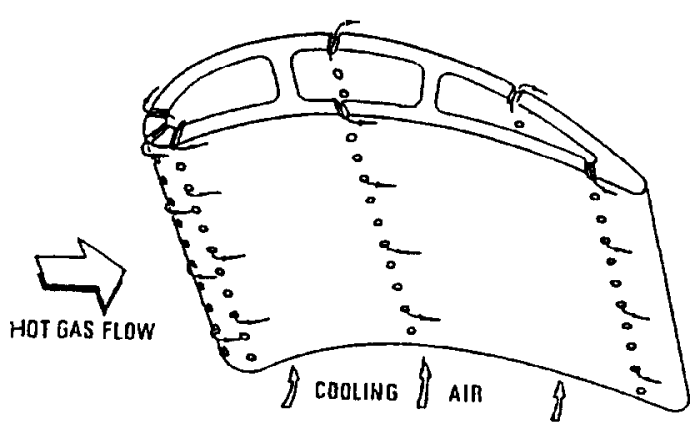

Fig 2. Film cooling system

exchange calculations for the prediction of the blade's metal temperature distribution with a combined cooling system ( $f$ g 3 ) and global cooling characteristics parameters.

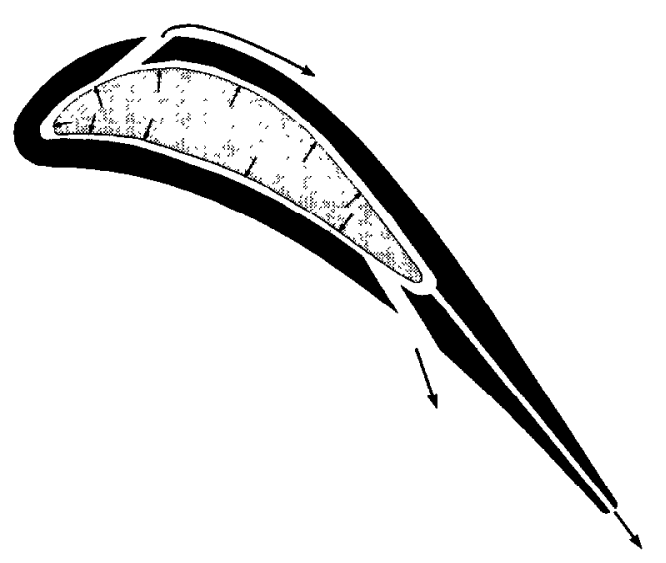

Fig 3. Compound cooling system scheme of the blade

\section{Global cooling characteristic parameters}

Global cooling characteristic parameters are:

- internal cooling efficiency: $\varepsilon_{h}$;

- global film cooling effectiveness $\eta_{f}$.

For a gas coolant, these are defined (Stecco and Facchini [16]) as:

$$
\begin{gathered}
\varepsilon_{h}=\frac{\left(T_{c e x}-T_{c}\right)}{T_{w}-T_{c}} \\
\eta_{f}=\frac{T_{a w}-T_{c}}{T_{c}-T_{f}}
\end{gathered}
$$

Internal cooling efficiency $\varepsilon_{h}$ shows the excellent capacity of the cooling system to remove the heat flux from the blade. The closer the exhaust coolant temperature $T_{c e x}$ is to the blade temperature, the better the coolant system. Global film cooling effectiveness $\eta_{f}$ shows how well the film cooling protects the blade from the hot gas heat flux; the blade temperature $\left(T_{a w}\right)$ is obtained when the blade is cooled by film cooling only with no other internal cooling system.

The coolant receives a heat flux which is equal to the heat flux produced by the hot gases. So, imposing total energy conservation and introducing medium external Stanton number, the coolant mass flow rate can be determined [16]:

$$
\frac{m_{c}}{m_{g}}=S t_{g} \frac{C_{p_{g}}}{C_{p_{c}}} \cdot \frac{\Omega_{c}}{\Omega_{g}} \cdot \frac{1}{\varepsilon_{h}} \cdot \frac{\left(T_{a w}-T_{w}\right)}{\left(T_{w}-T_{c}\right)}
$$

Equation (2) is valid for a generic cooling system, but it depends on the coolant and the cooling system used. To make out the internal cooling from external cooling, equation (2) can be also written as (Stecco and Facchini [16]):

$$
\begin{aligned}
& \frac{m_{c}}{m_{g}}=S t_{g} \frac{C_{p_{g}}}{C_{p_{c}}} \cdot \frac{\Omega_{c}}{\Omega_{g}} \cdot \frac{1}{\varepsilon h} \cdot \frac{\phi}{1-\phi}\left(1-\eta_{f}^{*}\right) \\
& \text { where } \quad \phi=\frac{\left(T_{g}-T_{w}\right)}{\left(T_{g}-T_{c}\right)}
\end{aligned}
$$

\section{STUDY OF VARIOUS COOLING MODELS}

\subsection{IMPINGEMENT}

The study of impingement is based on the works of Florschuetz et al [9]. They proposed a study of impingement on a flat surface, with no initial cross flow, and from there went on through the formulation of an equation to determine the exit flow velocity of the jets and of a correlation to calculate the Nusselt number. This study was continued and extended (Florschuetz and Isoda [10]; Florschuetz et al [11]) to include the case in which initial cross flow existed. Florschuetz and Tseng [12] proposed a study for a case in which the impingement geometry does not remain the same.

When trying to build a flexible model, one needs to extend the correlations taken from previous works to the most general case. In fact, the geometry of the impingement jets could only be briefly studied in the bibliography [9 to 12]. Therefore, because this study would like to be flexible and easily adaptable to the greatest number of stator blades possible, it was useful to go back to the differential equation governing the phenomenon and then integrate it, in the most general way possible, in order to apply it easily to a code. Therefore, the most general case is considered, in which the impingement model was made up of several zones with various geometries, and each geometry was characterized by the relationship $x_{n} / d, y_{n} / d, z / d$ (fig 4). 


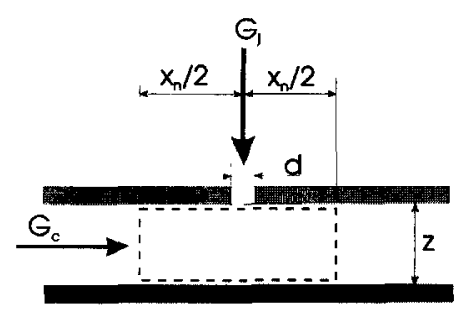

Fig 4. Injection model for impingement (Florschuetz et al [9])

The mathematical model used treats the holes as a surface with continuous injection $[9,10,12]$ so that differential notation can be used. The continuous injection can be shown as a case in which the jet passes through a porous material, thereby being continuous with respect to the $x$ direction.

Given the hypothesis of an incompressible fluid (a reasonable assumption since the flow velocity will usually be less than $M a=0.3$ ) and considering the friction losses, the differential equation of impingement in its complete form, which takes into account the equilibrium of the momentum, is written as:

$$
\mathrm{d} P=-\frac{2 \cdot G_{c} \cdot \mathrm{d} G_{c}}{\rho}-2 \frac{f \cdot G_{c}^{2} \cdot \mathrm{d} x}{2 \rho z}
$$

with the continuity equation, it can be written as:

$$
\frac{\mathrm{d}^{2} G_{c}}{\mathrm{~d} x^{2}}=M^{2}\left[1+f \cdot \frac{1}{2} \frac{G_{c}}{G_{J}^{*}}\right] G_{C}
$$

where:

$$
G_{J}=C_{D} \sqrt{2 \rho\left(P_{0}-P\right)} \quad M=\frac{\sqrt{2} A_{0}^{*} C_{D}}{z}
$$

and $f$ depends on the Reynolds number (Florschuetz and Isoda [10]). With respect to (5) and (6b) we will ignore only the variation of the flow coefficient $C_{D}$ as it varies in the relationship between the flow masses of the cross flow and the jet velocity [10].

We could also write:

$$
\frac{d^{2} G_{c}}{\mathrm{~d} x^{2}}-E^{2} G_{c}=0
$$

where:

$$
E^{2}=M^{2}\left[1+f \cdot \frac{1}{2} \frac{G_{C}}{G_{J}^{*}}\right]
$$

We can see that $E$ depends on the flow mass velocities of the cross flow $\left(G_{c}\right)$ and the jet $\left(G_{J}\right)$. The solution will then be:

$$
G_{c}=B_{1} \cdot \sinh E_{x}+B_{2} \cdot \cosh E_{x}
$$

Moreover, the mass velocity of each jet will be expressed by a similar expression:

$$
G_{J}^{*}=z E\left(B_{1} \cdot \cosh E_{x}+B_{2} \cdot \sinh E_{x}\right)
$$

This solution will have to be solved iteratively, because the coefficients $B_{1}, B_{2}$ and $E$ vary with the mass velocity $G_{c}$.

In order to solve (9), the problem must be discretized. Each control volume will then be examined as a separate zone and (9) will be written for each control volume, with the origin of the $x$ axis at the beginning of each one. The various terms are conventionally expressed with two indices $(i, j)$ indicating the $j^{\text {th }}$ control volume of the $i^{\text {th }}$ zone at constant geometry. Therefore, (9) and (10) can be expressed as follows:

$G_{C_{\imath, 3}}=B_{1_{i, j}} \cdot \sinh E_{i, j} x_{i}+B_{2_{\mathrm{i}, 3}} \cdot \cosh E_{i, j} x_{i}$

$G_{J_{2,3}}^{*}=$

$z E_{i, j}\left(B_{1_{\imath, j}} \cdot \cosh E_{i, j} x_{i}+B_{2_{i, j}} \cdot \sinh E_{i, j} x_{i}\right)$

In order to solve this problem, we must impose continuity on the pressure and on the mass flow rate among the various control volumes:

- interface boundary conditions between the various zones at constant geometry:

$$
\begin{cases}G_{C_{\imath k_{\imath}}}\left(x_{n_{\imath}}\right)=G_{C_{\imath}+1,1}(0) & i=1, n-1 \\ P_{i, k_{\imath}}\left(x_{n_{\imath}}\right)=P_{\imath+1,1}(0) & i=1, n-1\end{cases}
$$

- interface boundary conditions between the various control volumes within the same constant geometry zone:

$$
\begin{cases}G_{c_{i, j}}\left(x_{n_{\imath}}\right)=G_{C_{\imath, j+1}}(0) & j=1, k_{i}-1 \\ P_{i, j}\left(x_{n_{\imath}}\right)=P_{i, j+1}(0) & j=1, k_{i}-1\end{cases}
$$

- rearranging the boundary conditions by using (11) and (12) and writing them in matrix form, we have:

$$
\begin{array}{r}
\left(\begin{array}{c}
B_{1_{\imath}+1,1} \\
B_{2_{\imath+1,1}}
\end{array}\right)=\left(\begin{array}{cc}
K_{A_{\imath, \jmath}}^{I} & K_{B_{\imath, \jmath}}^{I} \\
K_{C_{\imath, \jmath}}^{I} & K_{D_{\imath, \jmath}}^{I}
\end{array}\right)\left(\begin{array}{l}
B_{1_{\imath, k_{\imath}}} \\
B_{2_{\imath, k_{\imath}}}
\end{array}\right) \\
i=1, n-1 \\
\left(\begin{array}{c}
B_{1_{\imath, \jmath+1}} \\
B_{2_{\imath, \jmath+1}}
\end{array}\right)=\left(\begin{array}{ll}
K_{A_{\imath}}^{I I} & K_{B_{\imath}}^{I I} \\
K_{C_{\imath}}^{I I} & K_{D_{\imath}}^{I I}
\end{array}\right)\left(\begin{array}{l}
B_{1_{\imath, \jmath}} \\
B_{2_{\imath, \jmath}}
\end{array}\right) \\
j=1, k_{i}-1
\end{array}
$$

where the $K$ constants depend on the geometry. By multiplying the matrices, the constant of the last 
control volume with respect to the first one can be determined:

$$
\left(\begin{array}{l}
B_{1_{n, k_{n}}} \\
B_{2_{n, k_{n}}}
\end{array}\right)=\left(\begin{array}{ll}
K_{A} & K_{B} \\
K_{C} & K_{D}
\end{array}\right)\left(\begin{array}{l}
B_{1_{1,1}} \\
B_{2_{1,1}}
\end{array}\right)
$$

The expressions of the integration constants can be substituted in the following boundary conditions:

- without initial cross flow:

$$
\left\{\begin{aligned}
G_{c_{1,1}}(0) & =0 \\
G_{c_{n, k_{n}}}\left(x_{n}\right) & =\frac{m_{J}}{z}
\end{aligned}\right.
$$

- with initial cross flow:

$$
\left\{\begin{array}{l}
G_{c_{1,1}}(0)=\frac{m_{c}}{z} \\
P_{1,1}=P_{i n i z} \Leftrightarrow G_{J_{1,1}}^{*}(0) \\
=A_{0_{1}}^{*} \cdot C_{D_{1}} \sqrt{2 \rho\left(P_{0}-P_{1,1}\right)}
\end{array}\right.
$$

Therefore, using the integration constants of the first control volume $\left(B_{1_{1,1}}, B_{2_{1,1}}\right)$, we can calculate the coefficients of the various regions, multiplying the matrices and determine the expression of the flow mass velocity inherent to the cross flow and the injected flow.

The calculations begin by setting $E_{i, j}=M$; now, with (8) the new value of $E_{i, j}$ can be determined and this calculation can be repeated until it converges.

Note that the viscosity $\mu$ depends on the temperature, and thus the entire calculation must also be iterated with respect to temperature.

Until now, only momentum and mass balance equations are used; to determine the flow temperature, energy equation is necessary:

$$
\Delta h_{0}=q X \Leftrightarrow \Delta h+\frac{\Delta u^{2}}{2}=q X
$$

To calculate the heat exchange coefficient, the correlations proposed in the above-mentioned papers (Florschuetz et al [9]; Florschuetz and Tseng [12]) are used. The analytic calculations determine a discontinuity in the heat exchange coefficient in the overlapping areas of the various geometries. However, it is important to point out that the greatest difference observed between the calculated value and the experimentally determined one in a nonuniform geometry is approximately $20 \%$ [12].

Like film cooling, impingement presents the problem of heat exchange between a wall and two flows (the cross one and the jet one). The influence of the two flows on local blade temperature is represented by means of the adiabatic wall temperature $T_{a w}$. In this case, $T_{a w}$ is the local blade temperature when the blade exchanged heat only with impingement. Generally, the adiabatic wall temperature is determined by using effectiveness, so that, with impingement, the effectiveness is introduced into the case where there is an initial cross flow (Florschuetz et al [11]):

$$
\eta=\frac{\left(T_{a w}-T_{J}\right)}{\left(T_{c}-T_{J}\right)}
$$

In this case, the use of an effectiveness term may not be appropriate because, in contrast with the film cooling, when greater effectiveness is better, with impingement, we want an effectiveness approaching zero when $T_{a w}$ is equal to $T_{J}$, and therefore to have the jet flow dominating.

\subsection{PIN FIN}

In the case of pin fins, we referred to the study of Armstrong and Winstoley [1] who proposed a synthesis of experiments on pin fins, thus offering some correlations for calculating the Nusselt number and the friction coefficient.

In this study, the correlations of Metzger et al [14] were used when the fins were short $(b / d 63)$ and those of Faulkner [8] when they were long $(b / d>3)$. The friction coefficient is determined from the Metzger correlations. In order to determine the physical state of the coolant flow after each row, one would need to keep track of the equation of continuity, the momentum, and the energy balance.

Because no fluid enters along the duct, the equation of continuity is:

$$
m_{c}=C t e \Leftrightarrow G_{c} \cdot A=C t e
$$

The static pressure loss in each row is (equilibrium of momentum):

$$
\Delta P=2 \cdot f \cdot \rho u_{\max }^{2}
$$

The overall enthalpy is increased through the heat given off by the blade (energy equation):

$$
\Delta h_{0}=q X \Leftrightarrow \Delta h+\frac{\Delta u^{2}}{2}=q X
$$

Assuming that air behaves as a ideal gas, we can use the corresponding law $(P=\rho R T)$. We have then four equations with four unknowns $(P, \rho, T, u)$. We can therefore reach a solution through an iterative method. Note that some parameters, such as the friction coefficient $f$ and the heat exchange coefficient $H$, depend on the Reynolds number, which in turn depends on the viscosity and, therefore, the temperature. It thus follows that the iteration must be done by updating these values too.

\subsection{TUBES}

The trailing edge can also be cooled, with obvious technological simplifications, by using a series of holes whose axes are parallel to the flow direction 
of the hot gases. Furthermore, film cooling holes can also appear like a series of tubes. Therefore, the code can handle tube cooling too, which can be studied using the continuity, energy, momentum and state equations, and the use of correlations for heat exchange which have been extensively discussed in literature (Necati and Özisik [15]). For the sake of brevity, the development of the equations will not be discussed, given their simplicity and the similarity to the treatment for pin fins.

\subsection{FILM COOLING}

Many experimental studies of film cooling have been carried out and it is difficult to summarize them. So we used a series of correlations proposed by L'Ecuyer and Soechting [13] for a study on film cooling, which determines the film's effectiveness by summarizing various published experimental results.

L'Ecuyer and Soechting have developed three different methods of injection: additional mass, mixing, and penetration. Effectiveness $\eta$ is determined by the PEP (Peak Effectiveness Parameter $\eta_{p}$ ) and its position $\left(\beta_{p}\right)$. These two parameters are calculated based on a series of graphs which show $\eta_{p}$ and $\beta_{p}$ as a function of $M_{f}$, of the ratio $u_{c} / u_{g}$, of the angle of injection and the dimensions of the equivalent throat $S$.

\section{DETERMINING THE MASS FLOW}

Blades of different gas turbines sometimes present cooling systems studied up to the present, but they are connected by different combinations, so that the global cooling system appears like a network. To study a global cooling system on a lot of blades, a modular code is necessary which must allow for the selection of the position and connection of each cooling system.

Until now, each type of cooling system has been examined separately from the others. One can see that each model is one-dimensional and requires the mass flow as input data, but, generally, the mass flow rates of each cooling system are unknown, only the total pressure upstream of the jet impingement and the static pressure at the point where the coolant comes out of the cooling system and mixes with hot gases are known. So, to determine the mass flow rate, the inlet and outlet pressure of each cooling system must be determined, too.

Each cooling system presents three unknowns and the equations used to solve this problem are:

- the flow mass of each model depends on the coolant pressure drop in each branch: for any cooling model $i$ it can be assumed that the pressure loss is roughly proportional to the square of the exit fluid velocity multiplied by the density (in a subsonic regime):

$$
P_{i_{\imath n \imath z}}-P_{i_{f \imath n e}}=c_{i}^{\prime} \cdot \rho u_{i}^{2}=c_{i} \cdot \frac{m_{i}^{2}}{\rho}
$$

For the case in which the density can be considered constant, the pressure loss can be reckoned proportional only to the square of the flow mass; an equation could be written for each of the cooling models;

- conditions of continuity of the flow mass and of the pressure in each node, which connects two or three cooling systems;

- in addition to the equations already written, the pressure upstream from the jets of the first models and the pressure in the coolant exit points of the blade are known.

Consequently, it is possible to completely simulate a generic cooling system by combining the various cooling techniques described with a onedimensional geometric domain.

\section{DETERMINING BLADE TEMPERATURE}

Through the distribution of the internal and external blade temperatures, the heat flow released by the hot gases to the blade and the heat absorbed by the coolant can be calculated. Because there are no sinks or heat sources in the blade cross section, the temperature will have to adapt so that the flow of entering heat (on the blade surface) is equal to the exit one (inside the blade). To determine the blade temperature, it is necessary to study:

- the internal heat exchange studied by the COOL code;

- the external heat exchange studied by the TRAF2D code;

- the conductivity in the blade cross section studied by the HT code.

\subsection{EXTERNAL HEAT EXCHANGE COEFFICIENT}

The distribution of the external heat exchange coefficient can be determined experimentally or with numerical models already developed and tested for this purpose. In order to simulate the external heat exchange coefficient of the blade, the TRAF2D code (Arnone, Liou, et al [2]) was used. In this code, the Baldwin-Lomax turbulence model and Runge-Kutta scheme to solve the NavierStokes equation are used. The Baldwin-Lomax turbulence model is simplified to the extent that the turbulence intensity level is taken into account only by imposing the transition point from laminar to turbulent boundary layer. 


\subsection{CONDUCTIVITY IN THE BLADE CROSS SECTION}

In addition to internal and external heat exchange calculations, a code studying the conductivity in the blade cross section will be necessary (Arnone et al [5]). The HT code was used to solve the two-dimensional steady heat equation (Fourier equation):

$$
\frac{\partial}{\partial x}\left(k \frac{\partial t}{\partial x}\right)+\frac{\partial}{\partial y}\left(k \frac{\partial t}{\partial y}\right)=q^{*}
$$

using a second-order finite differences method (Arnone and Sestini [4]). The HT code considers thermal conductivity variable, and corrected values of the material properties were defined to account for the presence of the film cooling holes and tubes. The thermal conductivity of sections with holes was evaluated as weight averaged between coolant and metal. The convection cooling effects of the film flow as it passes through the film holes is accounted for by heatsink $\left(q^{*}\right)$.

\subsection{INTERACTION BETWEEN CODES}

Using these three codes, the blade temperature can be determined. The calculation (fig 5) starts with an initial temperature distribution of the internal and external walls, which are input data in the COOL and TRAF2D codes. These supply the internal and external heat exchange coefficients and the adiabatic wall temperature. With this information, the $\mathrm{HT}$ code recalculates the temperature distribution on the internal and external surfaces of the blade, which allows the cycle to be restarted until it converges.

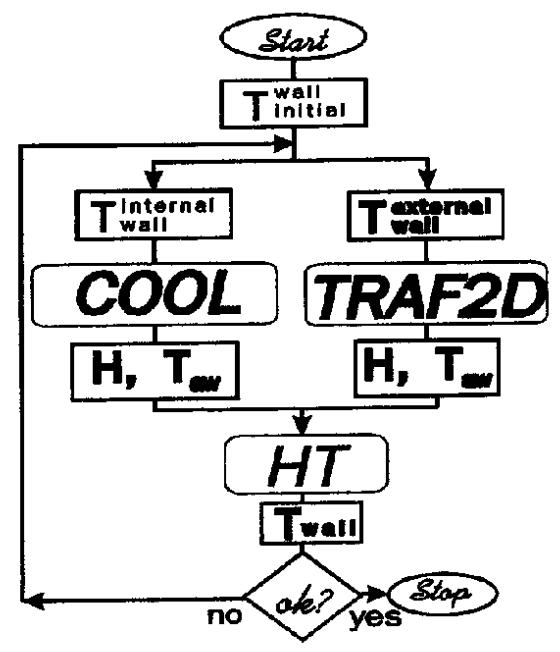

Fig 5. Flow chart calculation

\section{RESULTS}

\subsection{BLADE GEOMETRY}

In this study the blade reported on figure 3 , already evaluated by Arnone, Benvenuti et al [3], is analyzed. One of the nozzle blades studied is that of the PGT10 gas turbine, manufactured by Nuovo Pignone SpA.

Impingement, with various geometries, cools the internal blade. The exhaust coolant flow carries out film cooling on the suction side (near the leading edge) and on the pressure side (near the trailing edge). The trailing edge is cooled by tubes (fig 3 ). Figure 6 shows the global cooling system modeled.

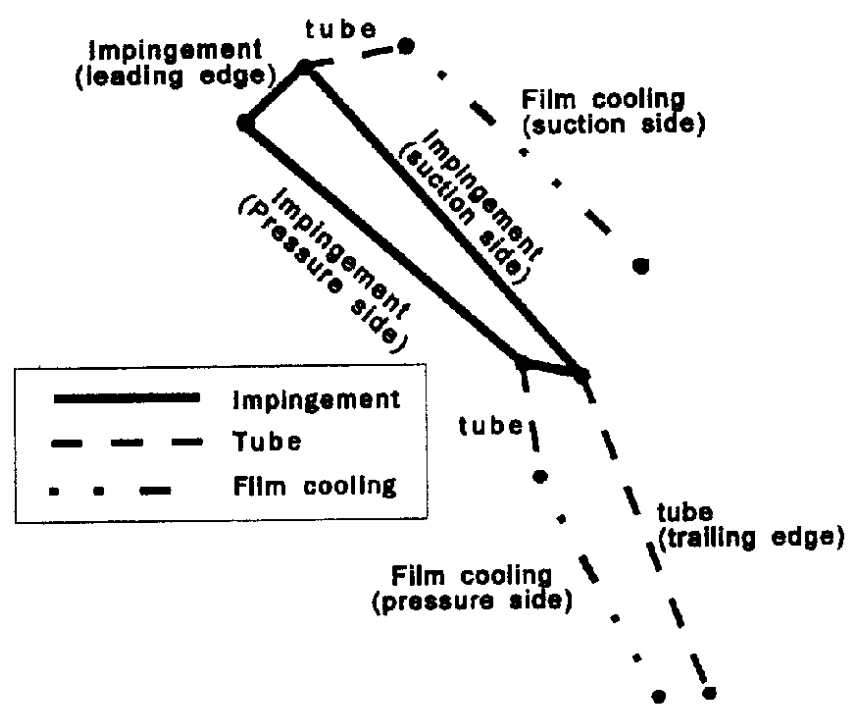

Fig 6. Modeling of the cooling system using one-dimensional models

\subsection{COOLING SYSTEM DESIGN}

The present study represents a useful and practical instrument to verify the temperature distribution in the stator blade cross-section and the response of the cooling system to the varying physical conditions of the coolant. It can also prove useful for the design of cooling systems in a stator blade.

The temperature distribution in the blade cross section is influenced by the blade geometry itself, by the distribution of the external heat exchange coefficient, and by the effects of internal and external cooling. In order to determine or check a certain temperature distribution in a blade, distinguishing these three points will help to determine where to increase or decrease cooling effects. 
To study the influence of blade geometry on the temperature distribution in the blade cross section, only a two-dimensional problem of heat exchange will need to be taken into account, assuming that the internal and external heat exchange coefficients are constant along the blade surface. For this calculation, only the HT code is needed.

On figure 7 we can see that the temperature peak is on the leading edge because of the typical blade geometry. In the blade mid-section, on both the pressure and suction sides, the distribution can be considered constant. On the suction side, a slight increase on the pressure side, due the unequal curvature, can be noted. At the point where the tubes begin, the temperature variation is sharp, because of the greater change in the thickness of the blade cross-section. Later on, the internal and external temperatures get closer to one another as the blade thins.

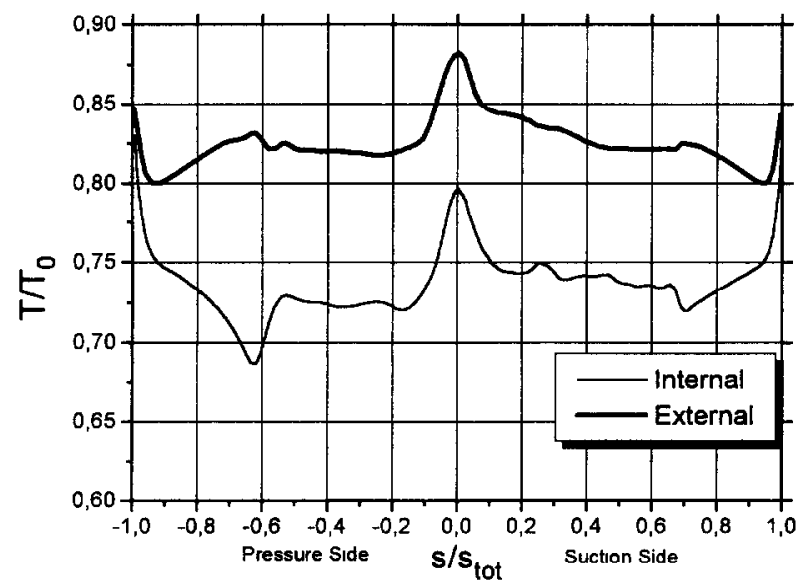

Fig 7. Blade temperature (blade geometry effects)

The influence of the external heat exchange coefficient can be observed by setting the adiabatic temperature distributions and the heat exchange coefficient on the inside of the blade constant. On figure 8 , an increase in the heat exchange coefficient on the suction side and at the end of the pressure side can be observed as a result of the greater hot gas flow acceleration and velocity. Instead, a low-heat exchange coefficient zone, coupled with a velocity value forms at the beginning of the pressure side. With respect to the previously mentioned curves, figure 9 shows an increase of temperature on the suction side and at the end of the pressure side where the heat exchange coefficient is higher because of larger hot gas flow turbulence. Instead, a low temperature zone forms at the beginning of the pressure side. Figure 9 shows which zones are especially delicate. On the leading edge, we have the combined effect of a high heat exchange coefficient and a blade geometry that does not ease up the work of the coolant. In addition, the suction side temperature is higher than the suction on the pressure side temperature which is mostly due to the lower external heat exchange coefficient with respect to the former. On the pressure side the temperature is shown to be high only at the edge.

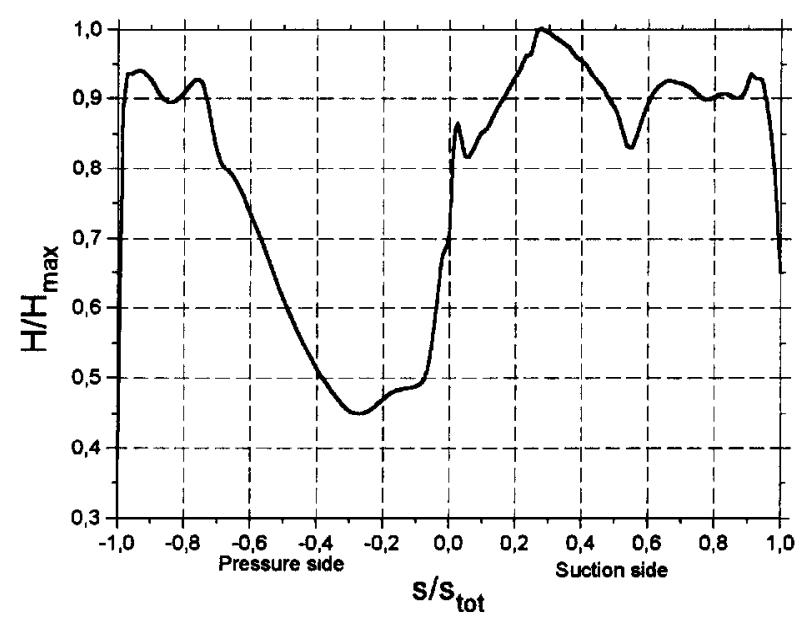

Fig 8. External heat exchange coefficient

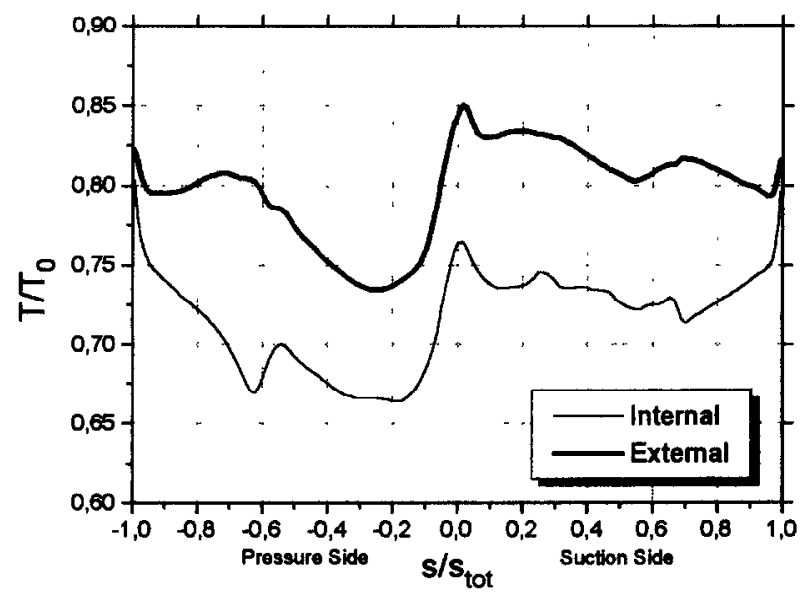

Fig 9. Blade temperature (blade geometry and external heat exchange effects)

From the observations made about the temperature influenced by blade geometry and the external heat exchange coefficient, some preliminary conclusions about the cooling system can be drawn. For example, the internal cooling system should be an impingement jet at the beginning of the blade where the heat exchange coefficient is high. In addition, the cooling system on the suction side will have to be more efficient than the one on the pressure side. Finally, we can see that the trailing edge of the blade must also be adequately cooled.

With the COOL code, we can determine the internal distributions of the heat coefficient and the adiabatic wall temperature of the cooling system shown on figure 3 (only internal cooling). On fig 10 , it is possible to see a high heat coefficient value at the leading edge of the blade. This is due to 
the high jet mass flow rate and the reduced mass cross flow. In other zones of impingement, the cross flow increases, reducing the jet mass flow rate and we have a heat coefficient decrease. With tube cooling it is possible to have a slight increase in the heat exchange coefficient, but in this case the adiabatic wall temperature, contrary to the effect of impingement, increases with coolant temperature $\left(T_{a w}=T_{c}\right)$. The temperature distribution in the blade cross section can be determined, when there is only one internal cooling system.

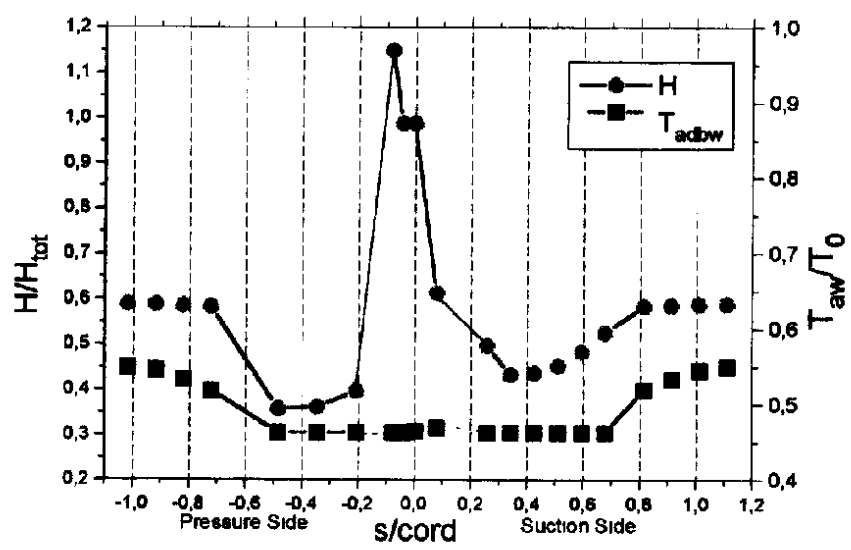

Fig 10. Internal heat exchange coefficient and adiabatic wall temperature

Figure 11 shows an elevated temperature along all the suction side and at the trailing edge of the blade on the pressure side. These results demonstrate that it is near the beginning of the suction side and at the end of the pressure side that film cooling has to be used. So, when redoing the calculations, the blade temperature distribution is obtained by taking into account the effect of film cooling. By analyzing the external blade temperature distribution (fig 12 and 13), we see that the maximum temperature is reached on the leading

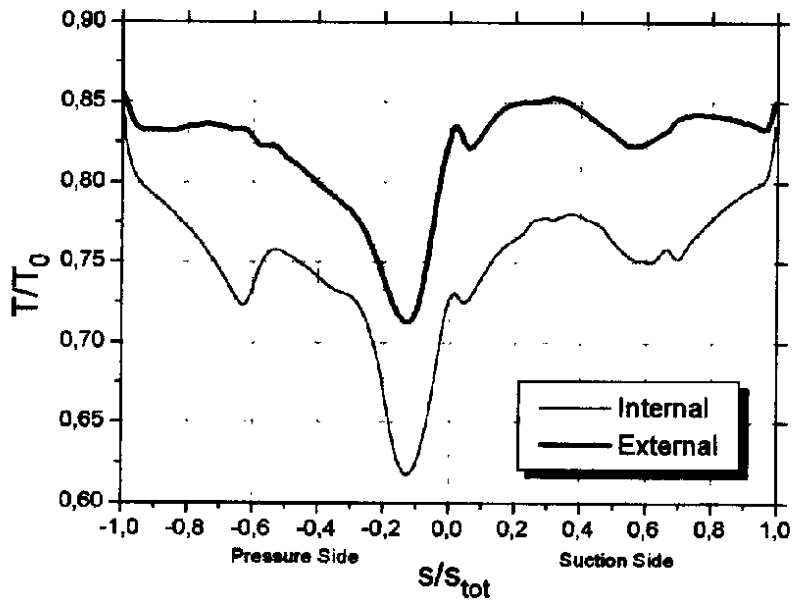

Fig 11. Blade temperature (without film cooling effects)

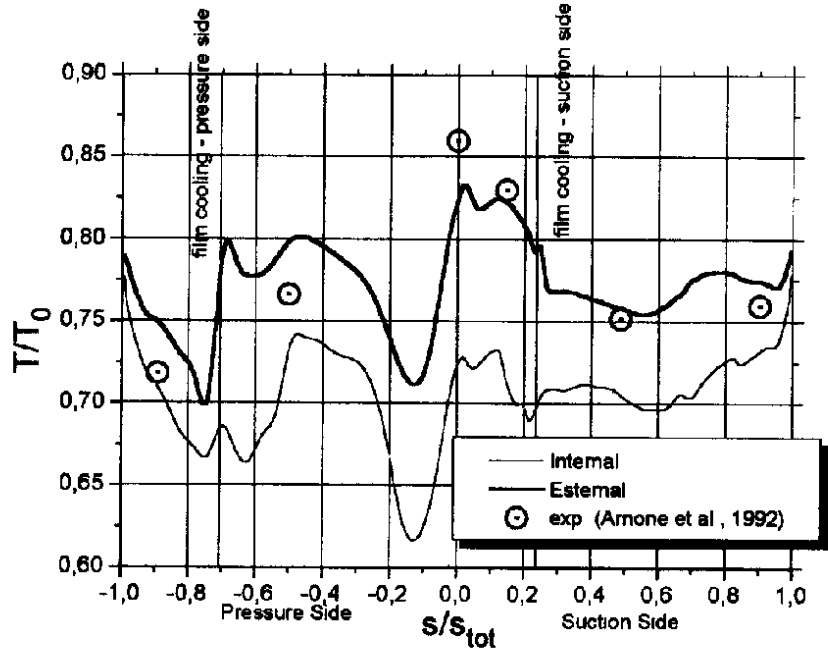

Fig 12. Blade temperature

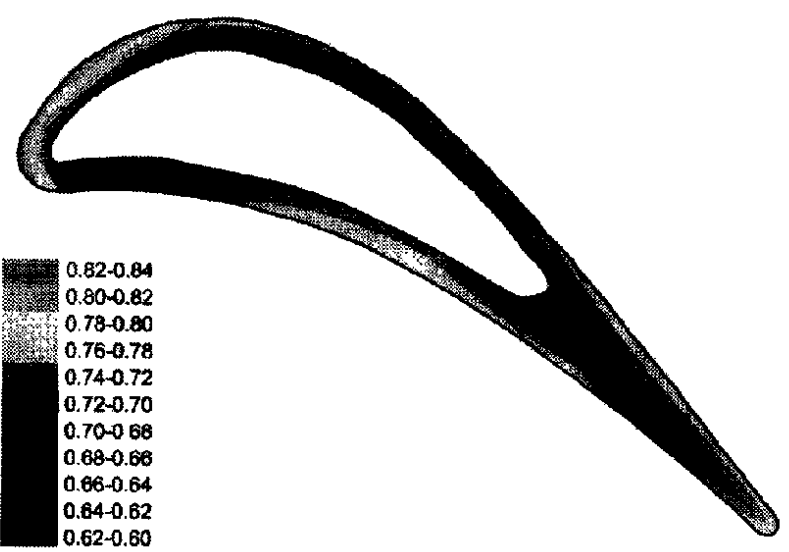

Fig 13. Blade cross section temperature contours

edge because it is not possible to compensate the negative effects of the high external heat exchange coefficient and the geometry with cooling. On the suction side, the temperature decreases rapidly at the point where film cooling begins. On the pressure side, the blade temperature is low because of the external heat exchange coefficient and the elevated cooling effect. Continuing towards the trailing edge, the temperature increases up to the beginning of the film cooling on the pressure side. The symbols on the figure are experimental measurements relative to the preceding research (Arnone, Benvenuti et al [3]) and show good agreement with the information available.

After the first calculations, the cooling system geometry can be modified for improvement. For example, if we notice a sharp lowering of the temperature at the beginning of the pressure side, we can reduce the effect of impingement in this zone and thus obtain a more uniform temperature distribution (fig 14). 


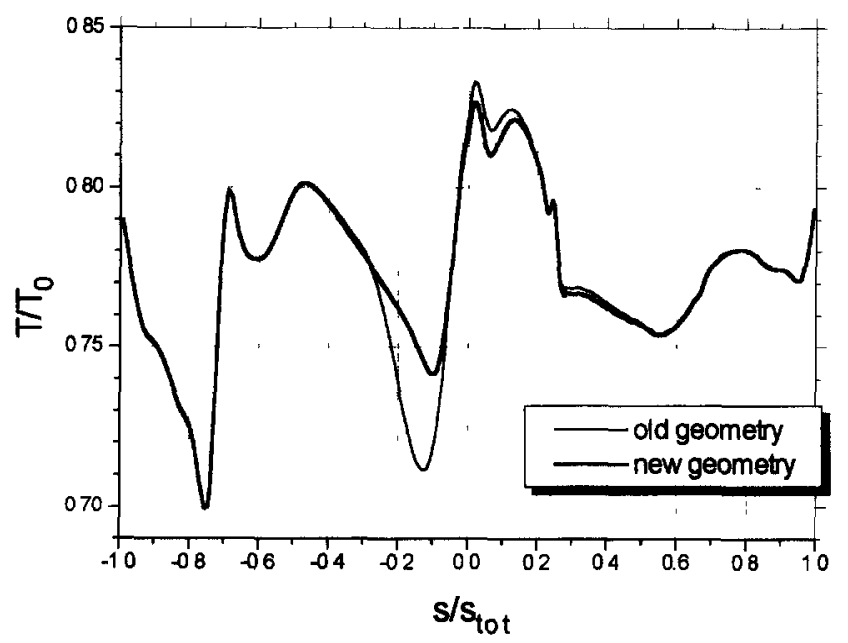

Fig 14. External blade temperature with different cooling geometry

On figure 14, we can see that by reducing the effect of impingement at the beginning of the pressure side, we obtain a smaller temperature gradient in the same area. In addition, a lower maximum temperature at the leading edge is another result. In fact, with the new cooling configuration, the cross flow due to impingement is reduced along the leading edge, with a subsequent increase in the cooling effect. With the solution proposed here, not only have the temperature gradient and the maximum temperature been lowered, but the mass flow of coolant has been reduced (about 5\%), thereby increasing the efficiency of the cooling system.

\subsection{BLADE COOLING SYSTEM OFF-DESIGN PERFORMANCE}

After the cooling system design, the off-design study is necessary. Coolant temperature and pressure at the inlet of the cooling system vary at partial loads conditions. Moreover, there is the possibility of limited modifications in the coolant characteristics (ie inter-refrigeration of coolant, or its bleeding at intermediate compressor pressure levels).

The modified coolant conditions correspond to a mass flow rate variation involving some effects on cooling efficiency and blade temperature distribution.

Figure 15 shows the total mass flow rate variation. It is possible to see a relevant coolant mass flow rate reduction with decreased coolant pressure; the dependence on total temperature is less consistent. The mass flow rate distribution between tubes in the blade trailing edge and the film cooling ducts on the pressure side is interesting: the mass flow rate in the tubes is almost constant, because the flow is near choked condition, while the film cooling ducts are designed to have a flow with low Mach number.

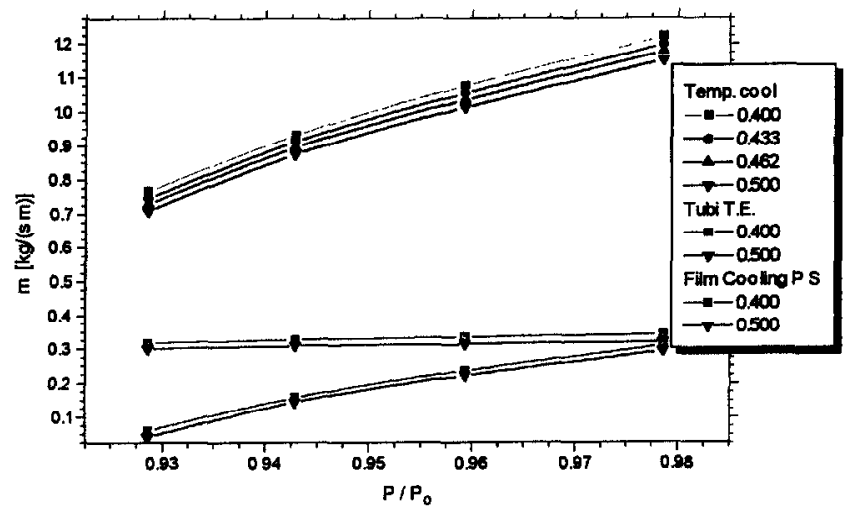

Fig 15. Coolant mass flow rate vs pressure and temperature of inlet coolant

The course of internal cooling efficiency (fig 16) follows an opposite trend: an increase in pressure corresponds to a decrease in efficiency because of a higher coolant flow rate and, if the mass flow rate is greater, the exhaust coolant temperature is less close to the blade temperature. In the contrast, an increase in coolant temperature determines a higher exhaust coolant temperature causing the efficiency to decrease.

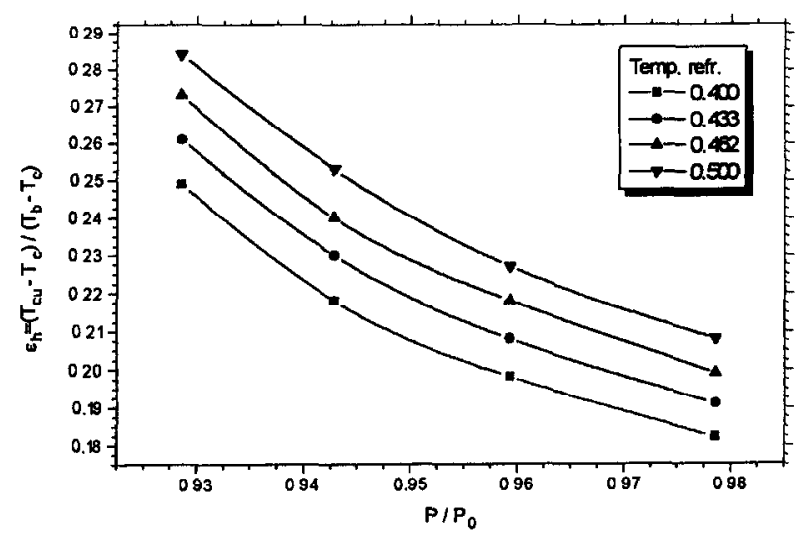

Fig 16. Internal cooling efficiency $\varepsilon_{h}$ vs pressure and temperature of inlet coolant

The global film cooling effectiveness (fig 17) presents a consistent increase with the pressure until the coolant mass flow rate becomes too high. The temperature effects are the opposite with respect to efficiency, because a lower coolant temperature allows higher efficiency.

Figure 18 shows the medium blade temperature variation. The medium temperature decreases with the pressure increase, because the mass flow rate increases too; and it increases with the inlet temperature increase. Figure 19 shows the course of the external blade temperature for three different conditions of pressure and temperature. 


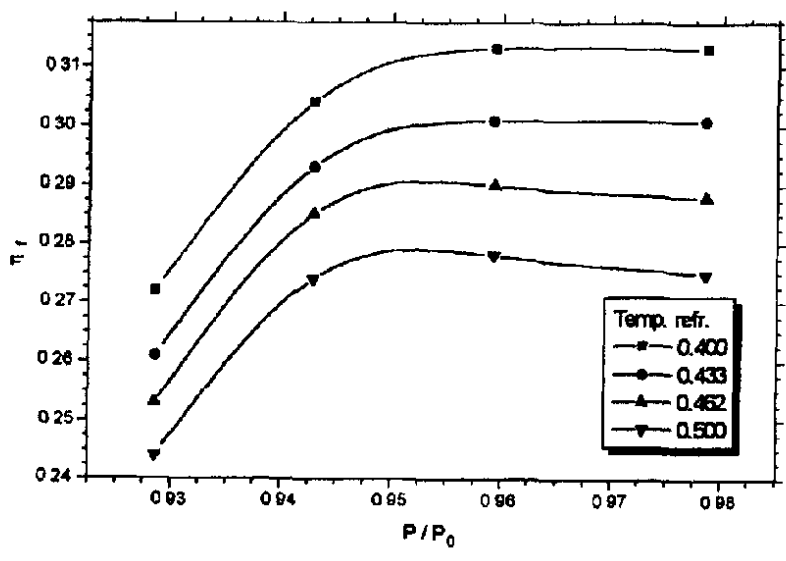

Fig 17. Global film cooling effectiveness vs pressure and temperature of inlet coolant

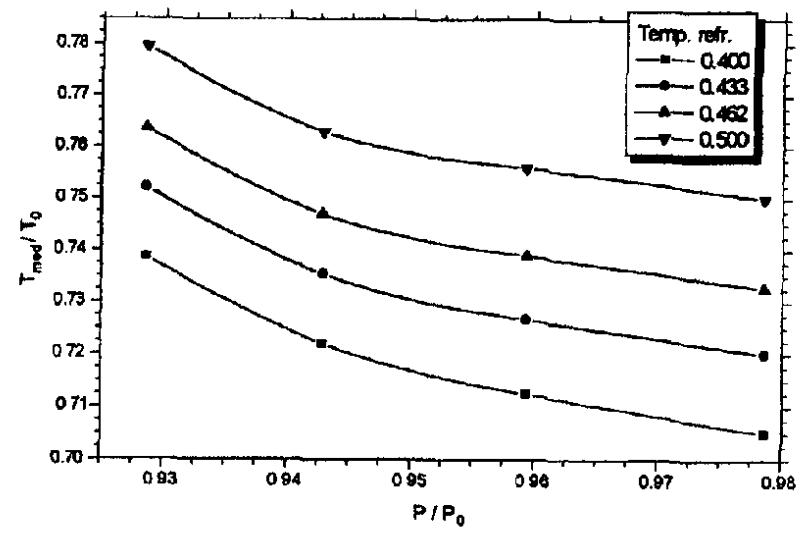

Fig 18. Medium blade temperature vs pressure and temperature of inlet coolant

\section{CONCLUSION}

A code has been proposed which can simulate the cooling effects on a stator blade in a turbine using a combination of different cooling techniques. By using a one-dimensional model, the coolant mass flow rate and the blade temperature distribution both on the internal and external sides can be calculated. In addition, the global heat exchange coefficients for each configuration were directly determined.

Following this, an application for the code was shown, proceeding to the analysis of the parameters that influence the blade temperature distribution, and showing good agreement between theoretical results and experimental ones. Finally, an example was shown to optimize the complete cooling system in order to obtain better temperature distribution across the blade cross section.

As far as the comparison with experimental results is concerned, the results agreed fairly well with the only test-case available, as long as the correlations widely validated with repeated and well-documented experiments were used.

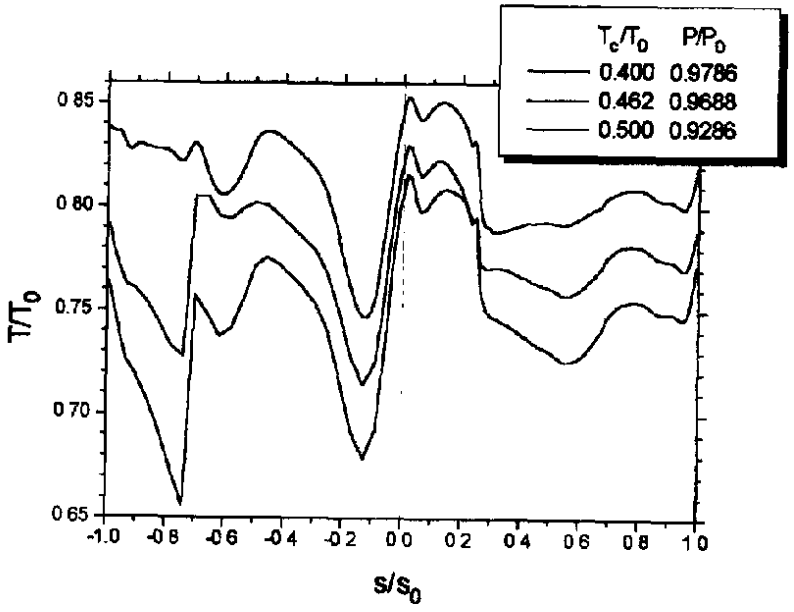

Fig 19. External blade temperature for three different pressure and temperature values of inlet coolant

Finally, the cooling system off-design performance was evaluated.

In conclusion, the COOL code and the method of studying cooling systems and blade temperatures are tools which make it possible to design a global cooling system and to test it in design and off-design conditions.

\section{Acknowledgements}

The authors would like to thank Pr E Carnevale and A Arnone for their valuable contributions to this work. They would also like to thank Nuovo Pignone SpA.

\section{REFERENCES}

[1]J Armstrong, D Winstanley (1988) A Review of staggered array pin fin heat transfer for turbine cooling applications. ASME J of Turbomachinery 110 , 94-103

[2] A Arnone, MS Liou, LA Povinelli (1992) Navier-Stokes solution of transonic cascade flow using non-periodic C-type grids. ASME J of Propulsion and Power 8, 2

[3] A Arnone, E Benvenuti, U Corradini, SS Stecco (1992) Theoretical and experimental investigation of temperature distribution in a gas turbine first stage nozzle. ASME COCEN-TURBO, 1992

[4] A Arnone, A Sestini (1991) Multigrid heat transfer calculations using different iterative schemes. Numerical Heat Transfer, part B: Fundamentals 19, 1.

[5] A Arnone, B Facchini, C Carcasci (1993) Valutazione e confronto degli effetti di alcune tecniche refrigerative nelle palettature statoriche di turbina a gas. VII Convegno Nazionale Gruppi Combinati, Milano, 21 22 October, 1993

[6] C Carcasci (1992) Tecniche di refrigerazione valutazione delle temperature nelle turbine a gas. Degree Thesis, DEF, University of Florence, 18 February 1992

[7] C Carcasci, B Facchini, U Corradini (1994) A numerical procedure to determine blade temperature 
of cooled stator blades in gas turbines: a numerical and experimental comparison. ASME COGEN TURBO POWER '94, Portland, Oregon-USA

[8] FE Faulkner (1971) Analytical investigation of chord size and cooling methods on turbine blade require ments. NASA CR-120882, 189-194

[9] LW Florschuetz, CR Truman, DE Metzger (1981) Streamwise flow and heat transfer distributions for jet array impingement with cross flow. ASME J of Heat Transfer 103, 337-342

[10] LW Florschuetz, Y Isoda (1983) Flow distributions and discharge coefficient effects for jet array impingement with initial cross flow. ASME J of Engineering for Power 105

[11] LW Florschuetz, DE Metzger, CC Su (1984) Heat transfer characteristics for jet array impingement with initial cross flow. ASME J of Heat Transfer 106, 34-41

[12] LW Florschuetz, HH Tseng (1985) Effect of nonuniform geometries on flow distributions and heat transfer characteristics for arrays of impinging jet. ASME J of Engineering for Gas Turbines and Power $107,68-75$

[13] MR L'Ecuyer, FO Soechting (1985) A model for correlating flat plate film cooling effectiveness for rows of round holes. Heat Transfer and Cooling in Gas Turbines.

[14] DE Metzger, WB Shepard, SW Haley (1986) Row resolved heat transfer variations in pin fin arrays including effects of non-uniform array and flow convergence. ASME Paper 96-CT-132

[15] M Necati, Özisik (1985) Heat Transfer: a basic approach. Mc Craw Hill, 317

[16] SS Stecco, B Facchini (1988) A simplified thermodynamic analysis of blade cooling effects in combined power plants. 2nd ASME COGEN-TURBO, Montreux, Switzerland

[17] SS Stecco, B Facchini (1989a) Refrigerazione palette di turbine a gas: effetti sul ciclo. Convegno Nazionale sui Gruppi Combinati, Bologna, Italy

[18] SS Stecco, B Facchini (1989b) Refrigerazione di palette di turbine a gas: determinazione dei parametri termofluidodinamici. Convegno Nazionale sui Gruppi Combinati, Bologna, Italy

[19] SS Stecco, B Facchini (1989c) A computer model for cooled expansion in gas turbine. 3rd ASME COGENTURBO Symposium, Nice, France 